\title{
Au temps \\ de la Société Royale de Médecine
}

> Le Rob Laffecteur s'est plaint, dans un monologue publié dans le numéro de mai 2013 de médecine/ sciences, des contraintes imposées par la Société Royale de Médecine en 1779 pour procéder à la labellisation des remèdes ${ }^{1}$. En même temps, il a su profiter de l'évaluation dans un but publicitaire, quitte à détourner l'esprit du rapport de son évaluation pour n'être présenté qu'à son avantage. La Société Royale de Médecine a été créée en 1778 pour traiter de tout ce qui avait trait à la santé publique. Active, séculaire et compétente, elle a embrassé nombre de problématiques auxquelles nous sommes confrontés de nos jours en matière d'évaluation des médicaments, en s'appuyant sur une évaluation scientifique rigoureuse reposant sur l'état des connaissances. <

\section{Le paysage de la santé publique en France à la fin de l'Ancien régime}

L'enseignement de la médecine avait lieu dans les facultés et collèges, et les facultés de médecine de Paris et de Montpellier dominaient.

Les médecins ne soignaient qu'une petite partie de la population, essentiellement citadine et aisée. Dans les campagnes, les soins étaient officiellement assurés par des chirurgiens, dont les compétences relevaient plus du parcours et des qualités personnelles des praticiens que d'une formation validée. La coopération entre médecins et pharmaciens, rendue nécessaire par le circuit de la prescription médicamenteuse, induisait une rivalité entre ces deux corps, les pharmaciens n'acceptant pas d'être de simples auxiliaires. Ceux-ci avaient plusieurs atouts: le contact avec le public, qui sollicitait leur avis, et leur aisance financière, qui n'avait rien à envier à celle des médecins dans de nombreux cas.

Vignette (Photo $@$ iNumis).

${ }^{1}$ Voir $\mathrm{m} / \mathrm{s} \mathrm{n}^{\circ} 5$, mai 2013 , pages $537-538$, en accès gratuit sur www.medecine sciences.org et directement via cette URL : http://www.medecinesciences.org/ articles/medsci/full_html/2013/06/medsci2013295p537/medsci2013295p537.html

\section{Pascale Gramain}

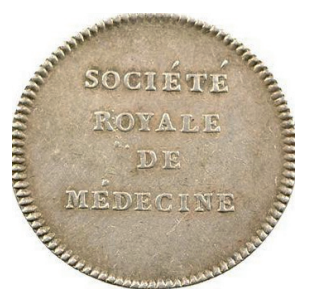

Docteure en histoire des sciences, ancienne auditrice de l'Institut des hautes études pour la science et la technologie (IHEST), Secrétaire générale du Cancéropôle Île-de-France, hôpital Saint-Louis, 1, avenue Claude Vellefaux, 75010 Paris, France.

pascale.gramain@canceropole$\underline{\text { idf.fr }}$

Enfin, les charlatans sévissaient en tous lieux, et sur toutes les catégories sociales. Ils étaient d'ailleurs très divers, allant de l'escroc à l'illuminé en passant par le rebouteux expérimenté ou le religieux dévoué. Un seul point leur était commun : leur activité était illégale. Les prescripteurs étaient, légalement, les médecins et les chirurgiens (pour ce qui est des remèdes externes). Les remèdes étaient prescrits le plus souvent sous forme de formules que préparaient les pharmaciens. Certains remèdes, qualifiés de «secrets», étaient des préparations vendues avec l'approbation des autorités - diverses de fait jusqu'à la mise en place de la Société Royale de Médecine (SRM) -, ou «à la sauvette » lors des foires notamment.

La législation sanitaire n'était que très incomplètement appliquée, mais elle connut des avancées remarquables avec la création de la SRM. L'existence de la SRM a représenté un indéniable progrès dans la prise en compte de la santé publique par le gouvernement, notamment sous l'angle de la validation des remèdes secrets.

\section{La Société Royale de Médecine}

\section{L'élite de la profession médicale}

La Société Royale de Médecine (SRM), créée par un arrêt du Conseil d'État du 29 avril 1776 et enregistrée au Parlement de Paris le $1^{\text {er }}$ septembre 1778, avait pour objet de s'occuper «de tous les faits de médecine théorique et pratique, et essentiellement de tout ce qui peut avoir rapport aux maladies épidémiques [...] et épizootiques ». Elle devint l'interlocuteur du gouvernement pour les problèmes de santé publique et, au-delà, la référence pour la nation. L'élite intellectuelle, à laquelle appartenaient les membres de la SRM, vit, dans les premiers temps de la Révolution, l'accomplissement des Lumières, une possibilité formidable de faire aboutir en pratique et rapidement des idées dégagées des contraintes de l'Ancien régime: «elle [la SRM] a cou- 
rageusement banni de l'art de guérir un despotisme qui n'était guère moins homicide que celui dont l'Assemblée nationale vient de nous délivrer ». La reconnaissance de cette fonction administrative était la première voie vers un ministère de la Santé à part entière.

La SRM était la structure la plus à même d'avoir une vision de la santé publique, du fait de ses relations avec les praticiens et de ses actions de terrain. Cette vision n'était sans doute pas exhaustive et comportait probablement de nombreuses lacunes, du fait notamment du manque d'outils statistiques, mais elle était la plus complète possible dans son contexte historique.

Vicq d'Azyr (Figure 1) en fut le secrétaire perpétuel depuis sa création. Médecin anatomiste célèbre, proche de l'Académie des sciences, travailleur acharné, il a été l'âme de la SRM; elle s'est pratiquement éteinte en même temps que lui. Elle fut en effet dissoute en 1793 comme toutes les sociétés savantes; il mourut en 1794 (le 2 messidor de l'an II). Le fonctionnement de la SRM était largement inspiré de l'Académie des sciences, dont plusieurs de ses membres faisaient partie. Mais elle fut en bute dès sa création à l'hostilité de la Faculté de médecine de Paris qui voyait en elle une concurrente et donc une menace pour son pouvoir.

La SRM était constituée de personnes, le plus souvent issues de la bourgeoisie, peu de la noblesse; quelques-unes avaient une origine modeste. Lorsqu'elles accédaient au second ordre, c'était par leur mérite ou leur fonction, et non par l'achat de charge («savonnette à vilains »). II n'y avait pas d'hégémonie parisienne parmi ses 179 membres associés : la moitié étaient issus de la province, et un tiers de l'étranger (médecins célèbres, surtout d'Europe septentrionale avec une ouverture au monde oriental). Plus de 400 correspondants étaient associés, dont une large majorité de médecins. Les membres, tous élus, constituaient l'élite de la profession médicale, ce qui leur autorisait une réelle autonomie intellectuelle, personne n'étant audessus d'eux dans leur domaine. Il est à noter que les critiques de leurs avis s'appuyaient sur des considérations non scientifiques.

Les assemblées de la SRM se tenaient deux fois par semaine sans exception ni vacance depuis 4 h et demie jusqu'à 6 h et demie. II y eut notamment une séance le 14 juillet 1789 au cours de laquelle les membres présents s'interrogèrent sur l'opportunité de continuer leur travail, et décidèrent de ne pas interrompre leurs activités, dans la mesure du possible, ce qu'ils firent effectivement.

\section{Une communication efficace avec le pouvoir, la presse, les praticiens}

Outre le compte rendu régulier de ses activités au roi (Louis XVI s'intéressait aux activités de la SRM, en augmenta les revenus et prit parti pour la SRM contre la Faculté de médecine de Paris), aux ministres et aux proches du pouvoir, il faut citer le rôle très important joué par le lieutenant général de police de Paris, Jean-Charles Pierre Lenoir (Figure 2). La SRM se tournait systématiquement vers lui pour tout ce qui concernait la police sanitaire. Il était aussi l'intermédiaire des ministres. Il n'hésita pas à s'impliquer personnellement en proposant des prix sur des thématiques médicales (qu'il subventionnait luimême), ou en obtenant des financements pour la SRM. II répondit aux
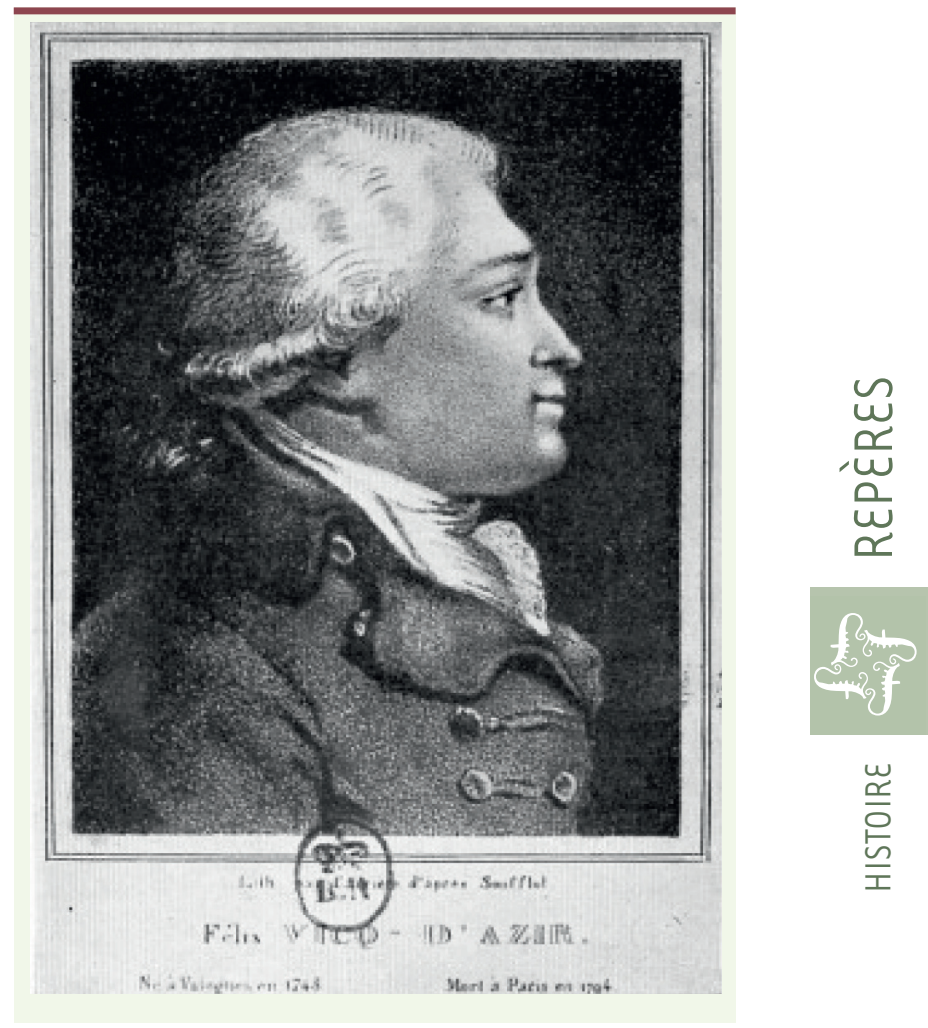

Figure 1. Félix Vicq d'Azyr (1748-1794), médecin et anatomiste. II n'existe pas à ce jour de bibliographie réellement complète, la somme de ses travaux étant considérable en quantité et en thèmes traités : anatomiste, il est l'un des pères de l'anatomie comparée. Membre de l'Académie des sciences, il fut envoyé par le gouvernement en 1775 pour endiguer une épizootie grave dans le sud-ouest de la France. À son retour, il organisa la Société Royale de Correspondance de Médecine, qui devint la Société Royale de Médecine dont il fut le Secrétaire perpétuel. II fut élu en 1788 à l'Académie Française (il a rédigé de nombreux éloges) et devint premier médecin de la reine Marie-Antoinette en janvier 1789.

demandes d'aide de la SRM pour lutter contre le charlatanisme et la publicité non contrôlée sur les médicaments, même s'il ne put résoudre l'immense problème des charlatans. L'importance que la SRM accordait à la communication trouva logiquement à s'exprimer dans le contrôle de la «publicité » faite aux médicaments qu'elle avait approuvés, et notamment dans la dénonciation des détournements des approbations (comme dans le cas du Rob Laffecteur). La SRM entretenait également des relations suivies avec les intendants en province.

Vecteur de diffusion, la presse n'était pas négligée par la SRM qui l'utilisait pour diffuser des informations (par exemple des listes de nominations) et veillait à ce qui paraissait dans les journaux. 
Enfin, elle s'appuyait sur son réseau français et international de praticiens qui envoyaient des mémoires en réponse à ses prix, ou qui la sollicitaient spontanément.

\section{L'évaluation et la labellisation des médicaments par la SRM}

Depuis Henri IV, les remèdes étaient approuvés par le premier médecin du roi. Dès le XvII ${ }^{e}$ siècle, des projets de création d'une sorte d’Académie de médecine pour évaluer les remèdes avaient tenté de voir le jour mais avaient été étouffés par la Faculté de médecine de Paris. Au milieu du XVIII ${ }^{e}$ siècle, la Commission royale de médecine était en charge de cette évaluation, mais n'avait pu fonctionner correctement, notamment du fait d'un manque de soutien fort du gouvernement.

La Société Royale de Médecine bénéficia en revanche de l'appui de Turgot, Necker, d'Alembert, Condorcet, Lavoisier et de celui de Louis XVI. Ses membres n'ont pas directement été à l'origine d'innovations thérapeutiques, mais ils ont organisé et mis en pratique ce qui avait été projeté - ou tenté - auparavant. Ils ont permis l'émergence de la science de l'évaluation des médicaments et, à terme, celle de leur découverte. En fait, l'examen méthodique des remèdes secrets devait permettre de se substituer au long travail de l'expérience du temps. La préoccupation de la qualité des règles instaurées par la SRM s'inscrivait dans deux logiques: d'une part être en conformité avec la science afin de permettre une expertise validée et témoigner d'une rigueur indispensable à la reconnaissance du monde scientifique et, d'autre part, asseoir l'existence administrative de cette compagnie par la qualité de son évaluation scientifique pour lui permettre d'être reconnue comme autorité irréfragable. Quelles étaient ces règles?

\section{Recevabilité du médicament par la SRM}

La société a défini ce qu'elle recherchait: des médicaments nouveaux quant à leur composition, leurs propriétés, leur préparation, leur administration. La condition sine qua non pour qu'un médicament soit examiné était donc que la recette ne soit pas connue (médicament non usité, recette non inscrite au codex), et en ait été communiquée à la SRM. Les commissaires examinaient si «elle peut remplir les promesses » que le possesseur annonçait, «si elle n'a aucun inconvénient pour la santé» (par une recherche «bibliographique »), et «si on peut en faire honnêtement l'essai ». La société utilisait ses compétences pour jauger celles des auteurs du médicament qui lui était soumis, et s'attachait à ne pas avoir d'a priori. Les certificats d'efficacité du remède étaient indispensables, mais ne remplaçaient pas la recette: «Des certificats nombreux ne peuvent pas être regardés comme un motif suffisant, puisque personne ne se présente, même avec le plus mauvais remède possible, sans en avoir recueilli un grand nombre ». Ils devaient être crédibles, et de préférence établis par des gens de l'art plutôt que par des particuliers : « cuisinier de Madame », vigneron, sacristain, postillon, marchand fripier, perruquier, qui certifiaient avoir été guéris. En fait, les possesseurs de médicaments « sérieux » soumettaient un dossier à la SRM. Ce dossier, pour être complet, devait comporter, outre la recette (composition, doses, manière de

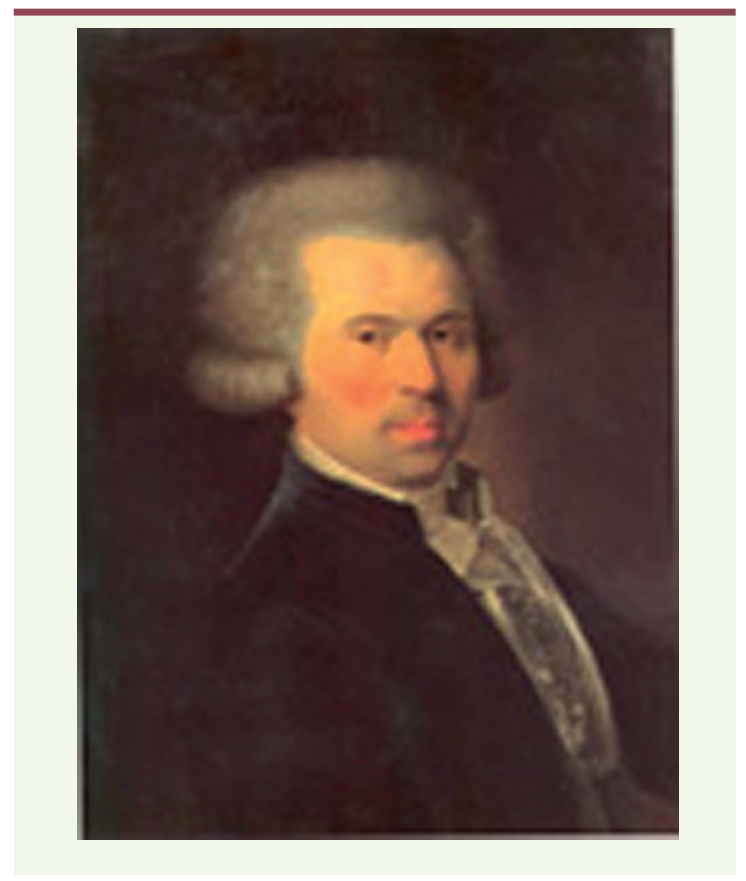

Figure 2. Jean Charles Pierre Lenoir (1732-1807), lieutenant général de police de Paris. «La police de Paris reçoit un nouvel éclat sous son administration ». Tous les établissements concernant la salubrité, l'approvisionnement, la bonne tenue des halles et des marchés, l'arrosement, l'éclairage, les incendies, les secours publics lui doivent de nombreuses améliorations. Le Mont-de-Piété lui doit son existence. «II emploie la répression contre la mendicité, la prostitution, les maisons de jeux avec la mesure convenant au temps et aux circonstances ». Il a rédigé ses mémoires à la fin de sa vie (archives d'Orléans).

préparer), des témoignages de l'efficacité du médicament et, enfin, l'auteur devait indiquer dans quel cas son remède était utile.

Les motifs de rejet étaient nombreux: absence de nouveauté (214 cas) ; caractère confus de la recette (58 cas) («chaos informe», «monstruosité pharmaceutique ») ; inefficacité, soit que les propriétés soient improbables, l'inefficacité évidente (62 cas) («substances se détruisent mutuellement», «connu par tous les médecins comme de très petits moyens »), ou la dangerosité ( 80 cas) supposée par la composition («poison très actif», «nous ne doutons pas de leur efficacité mais la substance qui les composent sont si dangereuses qu'à peine la verrait-on sans effroi entre les mains même des meilleurs médecins ») ou par le retard à l'utilisation d'autres méthodes plus efficaces («l'empêcher puisque en en faisant usage les malades peuvent perdre un temps qu'ils emploieraient plus utilement s'ils confiaient leur cure aux personnes en état de la diriger »). 
Conduite des essais : la nécessité de l'expérience

La nécessité de l'expérience a été imposée dès la première moitié du XVIII ${ }^{e}$ siècle par les philosophes. Au XVII siècle, des expériences médicales sur des médicaments avaient déjà lieu : «Nous avons montré qu'on pouvait mêler des médicaments aux liqueurs de notre corps pour voir l'effet qu'ils produisaient [...] quantité d'autres médecins avaient commencé avant lui cette manière de guérir, particulièrement quelques anglais, mais comme elle semble hasarder la vie des hommes, ils ne la faisaient d'ordinaire que sur les animaux. Et ils firent plusieurs belles observations qui pourront servir dans la suite à la guérison des maladies. Car l'on peut dire qu'on n'a pas fait encore assez d'expériences pour se servir de cette façon de seringuer les médicaments dans le sang; ainsi l'on ne la permettrait jamais, qu'en des maladies désespérées et où l'on ne pourrait souvent retirer aucun fruit. »

Ce texte induit une distinction entre des raisons méthodologiques et éthiques à la mise en place des essais. Si les raisons méthodologiques sont évidentes, celles qui ressortent de l'éthique sont plus nuancées, car, à peine refuse-t-on la moindre prise de risque («semble hasarder ») que la pratique est de fait opérée sur des humains, la rigueur affichée est donc suivie, en général. Une distinction peut donc être opérée entre ce qu'il conviendrait idéalement de faire, suivant des principes hauts placés, ce qu'il est acceptable de faire, et ce qui est finalement fait. Le flou de ces frontières amène à envisager les essais au cas par cas, ce qui ouvre la porte à des interprétations dont la motivation peut être d'ordre varié (éthique, scientifique, thérapeutique, financier, etc.).

Dans la perspective néo-hippocratique dans laquelle la SRM se situait, l'expérience étayait l'observation. Dans cette optique, les essais permettent de devancer l'expérience du temps. Les témoignages ne sont en aucun cas suffisants pour faire approuver les médicaments: si la SRM jugeait le remède potentiellement intéressant, elle tentera des essais de toute façon avant de l'approuver: «toutes les assertions du sieur Dubarbier ne peuvent rien contre le témoignage de l'expérience ». La SRM fera donc sien le principe de la nécessité de l'expérience, car elle avait pour but de pouvoir affirmer sans conteste l'innocuité et l'efficacité des médicaments.

\section{Définition des critères méthodologiques}

«Les essais doivent être faits avec la plus grande précaution, et de manière que l'on ne puisse en infirmer l'authenticité ».

La SRM mit en place une procédure de nomination des commissaires chargés d'examiner les recettes soumises à la SRM qui ne devait pas pouvoir porter le flanc à la critique et préservait l'indépendance des commissaires : «M. de Jussieu ayant représenté à la société qu'il serait à propos, lorsqu'on nomme des commissaires pour différents objets, de ne jamais choisir parmi les membres de la société ceux qui connaissent ou qui sont du même pays que les auteurs des mémoires envoyés, la compagnie a adopté cet avis. »

Elle entreposa les médicaments à tester en lieu sûr, «on a été aux voix pour savoir s'il convenait que le rob qui doit servir au traitement des six malades pour les nouvelles expériences fut déposé chez un des commissaires nommés pour suivre les effets. La société a pensé

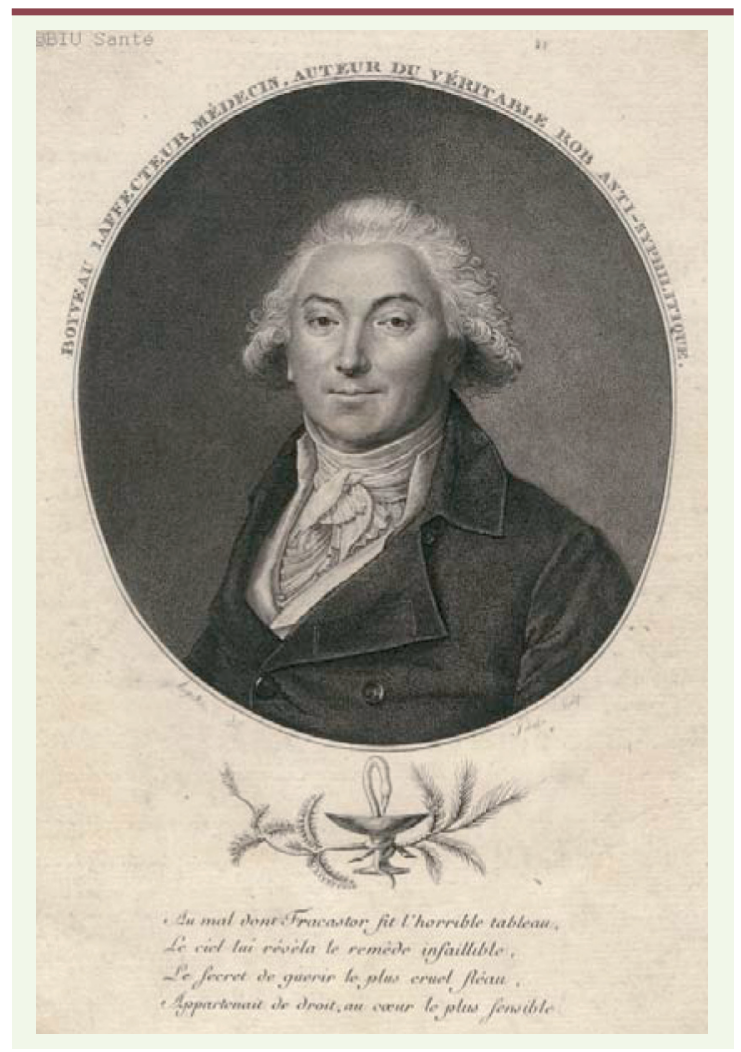

Figure 3. Pierre Boyveau (1743-1812), chirurgien ou pharmacien selon les sources, puis, semble-t-il médecin. II conservera le nom de Boyveau-Laffecteur, puis uniquement de Laffecteur pour ses affaires. En effet, lié par mariage à la famille de Marcilly, noble, il ne devait pas entacher le nom de son épouse dans des transactions commerciales. Denis Laffecteur : inspecteur des vivres au ministère de la Guerre, prête-nom rémunéré de Pierre Boyveau.

que cette liqueur devait être enfermée en l'endroit même où seront les malades dans une armoire à quatre serrures dont chacun des commissaires aura la clef et que lorsqu'un d'eux ne pourra se trouver à l'heure indiquée pour administrer le rob, il enverra la clef à l'un des trois autres commissaires », s'assura que le médicament testé était bien le même que celui proposé par l'inventeur en constituant deux lots de malades: un groupe traité avec le médicament préparé par les commissaires, et l'autre avec celui préparé par l'inventeur «afin de faire la comparaison de leurs effets réciproques».

La SRM conviait tous les médecins à assister à l'essai. Laffecteur (Figure 3) déplora que peu de médecins aient assisté à des essais sur son médicament [1] (voir Encadré) $(\rightarrow)$ : «il aurait été avantageux pour moi que

$\rightarrow)$ Voir $m / s n^{\circ} 5$, mai 2013, page 537 leurs affaires leur eussent permis de se rendre quelque fois à la visite qui se faisait régulièrement dans le dépôt 


\section{SYPHILIS ET MERCURE}

L'affaire du rob Laffecteur a eu autant d'ampleur parce qu'elle touchait le mal vénérien, redoutable fléau qui était à la croisée de la santé publique, de la morale, des forces vives de la Nation.

On ne sait pas au juste quand la syphilis est apparue en Europe. On a longtemps incriminé le Nouveau Monde, mais des traces antiques de syphilis ont été détectées sur le vieux continent. En revanche, il est certain que cette maladie était ressentie comme mystérieuse et effrayante.

Parmi les remèdes proposés, le mercure en onguents et pommades resta la thérapeutique quasi officielle pendant quatre siècles. On ne connaît pas l'origine de cette utilisation : une piste pourrait être l'utilisation du mercure contre la lèpre, autre maladie objet de fantasmes et que caractérisent des manifestations dermatologiques, comme la syphilis.

Les effets toxiques redoutables du mercure ont été identifiés rapidement (stomatite, gastrique, alopécie, etc.), mais le préjugé sur son efficacité (discutable en réalité) était enraciné dans la société depuis des siècles. Aucune alternative thérapeutique n'existait, mais de nombreux remèdes étaient proposés dans le but de concurrencer le mercure, dont la salsepareille (anti-inflammatoire, diurétique, sudorifique) qui entrait dans la composition du rob Laffecteur. On sait depuis Nichols (1912) que le mercure ne tue pas les tréponèmes. À partir de 1943, les malades furent traités par la pénicilline, et l'utilisation du mercure dans cette indication a été de fait abandonnée.

où les malades ont été traités; mais parmi les médecins qui ont signé les procès verbaux, tous à l'exception d'un seul, sont membres de votre compagnie ». L'inventeur du médicament ne devait pas savoir où se déroulait l'essai, du moins en théorie «dans un lieu sûr et ignoré du sieur Laffecteur ». Dans un essai ultérieur, la SRM adopta une attitude différente : «[...] qu'il serait avantageux d'inviter l'auteur à y assister, et à faire lui même l'application de son eau. »

Ces deux attitudes semblent obéir à deux objectifs différents: si les possesseurs n'assistent pas aux essais, ils ne peuvent les influer, s'ils y assistent, ils ne peuvent les critiquer.

Enfin, la SRM prenait en compte la pertinence par rapport aux autres essais en cours, évitant les duplications, et estima parfois qu'il fallait tester les médicaments en usage.

Échaudés par l'affaire Laffecteur, les membres de la SRM délibérèrent en 1782 : «souvent les rapports envoyés au ministre étaient communiqués aux particuliers dont les remèdes se trouvaient désapprouvés; ce qui occasionnait de leur part des réclamations et même des mémoires injurieux contre les auteurs de ces rapports. [...] La compagnie a arrêté que dorénavant on n'enverrait au ministre que les conclusions de ces rapports en forme de délibération de la société : qu'ils ne seront envoyés en entier que dans le cas où le roi ordonnerait expressément qu'on les lui communiquât; et qu'alors il serait chaque fois représenté au ministre et au roi, notre auguste protecteur, combien il est important que les personnes intéressées ne connaissent pas les noms de ceux qui ont été commissaires des objets qu'ils ont présentés. »

\section{Critères éthiques}

\section{Quelle justification, quel responsable pour l'essai ?}

La SRM affirma qu'il ne pouvait exister de passedroit: l'absence de recette interdisait à la SRM de tenter des essais et d'approuver le remède, y compris si l'inventeur était appuyé au plus haut niveau. Pour cela, elle s'en tint au respect rigoureux des lois: «le ministre a remis deux procès-verbaux dressés d'après des expériences faites sur ce remède, on a été aux voix et la société a arrêté que ce remède paraît devoir être soumis à la règle prescrite, que la société ne peut approuver une préparation qu'elle ne connaît pas et que les commissaires nommés pour tout ce qui a rapport aux empiriques dresseront un mémoire qui sera présenté au ministre pour lui représenter combien il serait dangereux de s'écarter de l'esprit des lettres patentes pour un remède dont le brevet en détruit par l'article $X$ des mêmes lettres patentes. »

Et pourtant, nous avons trouvé des exceptions... qui laissent supposer que la SRM a fait l'objet de pressions, sans pouvoir déterminer de quel ordre, sauf à nous contenter de motifs «politiques», terme pris ici dans son sens péjoratif.

\section{Le choix des patients : l'exemple du Rob Laffecteur}

Les essais menés par la SRM étaient toujours des essais sur l'homme (en 1790, Vicq d'Azyr insista sur le nécessaire rapprochement des médecines humaine et vétérinaire). Compte tenu du risque inhérent à ces tests, les essais sur les condamnés ont été envisagés, mais récusés. Même si la SRM pensa que c'était au pouvoir de décider - elle n'entendait pas s'y substituer, prenant garde à ne pas outrepasser ses droits -, elle affirma néanmoins qu'on ne pouvait procéder à des expériences sur les criminels. On ne pouvait leur infliger de peines supplémentaires, bien qu'ils aient été condamnés à mort, car si on les utilisait pour des expériences, «comme la loi a proportionné les supplices aux crimes ils endureraient un châtiment porté au delà de la punition qu'on a cru devoir leur infliger, ce qui serait barbare et anticonstitutionnel. »

Cependant, en 1779 , la société envisagea très sérieusement de faire des essais du rob Laffecteur sur des syphilitiques détenus de force à Bicêtre.

Amelot (Figure 4) informa Vicq d'Azyr le 18 octobre 1879 qu'il avait reçu une lettre " au sujet des expériences que la société désire faire du remède du sieur Laffecteur sur des personnes détenues de force et qu'on puisse retenir encore pendant un an après le traitement. Je viens d'en écrire à $M$. Lenoir et je le prie de faire tout ce que les circonstances pourront permettre. » 
Lenoir s'y opposa fermement (23 octobre 1879) : « il est absolument impossible, Monsieur, de livrer des personnes détenues de force, au traitement du sieur Laffecteur. Beaucoup de motifs s'y opposent et d'abord on trouverait difficilement à Bicêtre douze prisonniers attaqués de cette maladie, attendu qu'on a le soin de leur faire passer des remèdes, s'il en ont besoin dès qu'ils sont retenus en l'hôpital. D'ailleurs il me semble que l'on ne doit pas contraindre un malheureux à l'épreuve d'un remède. On doit consulter la volonté de ceux qui s'y soumettent. Les gens détenus à Bicêtre sont tous des scélérats, ou des jeunes gens renfermés pour mettre à l'abri l'honneur de leur famille; s'ils viennent à s'évader, ou à se procurer leur liberté, comme il leur sera plus facile qu'à Bicêtre, jugez des conséquences. Enfin les retenir de force dans une maison particulière un an après le traitement, serait une espèce de chartre privée, tant de motifs et d'autres que je pourrais relever s'opposent au parti proposé. »

Cette très belle lettre est importante car elle montre que les Lumières sont entrées dans la pratique professionnelle des hommes d'administration:

- les personnes ne peuvent être prises parmi les prisonniers car ceux-ci sont soignés dès qu'ils sont malades;

- la notion de consentement des personnes pour essayer des remèdes n'est pas sans rappeler nos lois modernes (loi Huriet de 1988);

- le respect de la personne quelle qu'elle soit, y compris si elle appartient à la lie de la société.

Lenoir réagit d'abord en tant qu'homme des Lumières et respecte l'individu, alors qu'on aurait pu supposer que sa fonction le prédestinait plutôt à appliquer avant tout et en toute circonstance la loi, sans émettre d'avis.

La SRM avançait une autre raison pour le choix de prisonniers: la priorité de la SRM était la santé de la population et donc la qualité des médicaments autorisés. Pour assurer cette qualité en termes d'innocuité et d'efficacité, le déroulement des essais devait être irréprochable. II importait donc de s'assurer que les malades ne pourraient contracter de nouveau la maladie dans le temps de la guérison, et devaient pouvoir être suivis assez longtemps (éviter les «perdus de vue »). Les prisonniers pouvaient représenter une population de choix car enfermée.

Les malades choisis étant tous très atteints et sans espoir de guérison par les remèdes habituels, on peut supposer qu'ils étaient d'accord pour tenter de nouveaux médicaments, étant donné la possibilité d'être traité par les meilleurs médecins du royaume, et en bénéficiant de conditions de vie a priori meilleures qu'à Bicêtre. Accord implicite de surcroît en raison du rapport d'obéissance que peut entretenir une personne dans un environnement institutionnel (hôpital ou caserne) vis-à-vis de sa hiérarchie. Lenoir ne soulève le problème que pour les prisonniers, car ils n'ont pas librement accepté ce rapport hiérarchique avec l'administration pénitentiaire.

Lorsque les essais furent mis en place par la SRM, les commissaires se réservaient le droit de choisir les malades qui devaient répondre à des critères bien définis, et être de préférence disponibles et suffisamment asservis pour être contrôlés. La préférence allait aux malades «naïfs » (c'est-à-dire n'ayant pas encore été traités), récemment affectés,

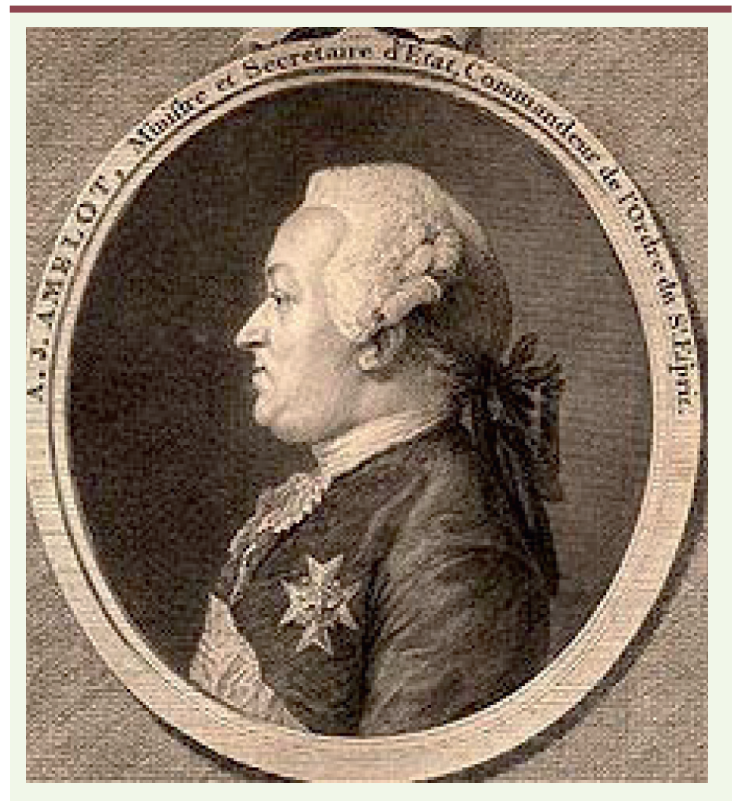

Figure 4. Antoine-Jean Amelot de Chaillou (1732-1795). Secrétaire d'État de la Maison du Roi avait sous sa direction l'administration de Paris et des provinces, les Affaires générales de la religion, les Bénéfices, les Dons et brevets, et l'administration de divers pays. Amelot suivait donc l'administration de la police de Paris, les lettres et des placets adressés par les particuliers, ainsi qu'un certain nombre de dossiers de procès dont les brevets, états et comptes des charges et des dépenses de la Maison du Roi.

sans pathologie associée; l'isolement des malades était considéré comme un élément essentiel, car il réduisait les paramètres qui pouvaient influer la santé.

\section{Conclusion}

La Société Royale de Médecine a analysé et examiné environ 500 remèdes de sa création à 1783 , et 442 demandes du 17 octobre 1780 au 21 juillet 1789 . Mais quelle a été son influence sur les pratiques médicales? II ne semble pas possible de l'estimer. En effet, nous n'avons réuni que peu d'ordonnances pour le XVIII ${ }^{e}$ siècle. Très peu de remèdes ont été approuvés (les nombres varient selon les documents, de quatre à une dizaine, il existait différents types d'approbation).

Qui, de la société ou de la SRM, a influé sur l'évolution de l'autre? Lumières, Révolution, instances d'évaluation des médicaments, préoccupation de la santé publique, les liens sont complexes.

Un élément de réponse est fourni si l'on considère que les bases étaient posées depuis longtemps. La commission de Sénac (président de la Commission de médecine au milieu du XVIII ${ }^{e}$ siècle), par exemple, disposait des 
arguments et règlements nécessaires à un fonctionnement honnête et efficace, mais dévia (il a été accusé par Grimm de vendre les approbations de remèdes). C'est donc une question d'hommes et de volonté politique. Les Lumières, filles des raisonneurs du Grand siècle, ont influencé les classes dirigeantes qui, à l'aube de la Révolution, ont mis en place la SRM. Ces considérations nous invitent à méditer sur la place de l'Histoire, à la nécessaire humilité qu'elle induit et qui replace la SRM dans une évolution de la société : la SRM a pu s'exprimer sur un terrain préparé depuis des décennies comme le montre l'historique des institutions d'approbation des médicaments. Ses membres fondateurs ont su exploiter des conditions favorables et en tirer le meilleur parti, ce que d'autres, peut-être, n'auraient pas su faire.

Nous assistons bien, à partir de la fin de l'Ancien Régime, à la construction d'une science : celle de l'évaluation des médicaments. La compétence, qui est alors requise, tend à la professionnalisation de ses détenteurs, qui peuvent, et c'est ce qui est vivement reproché à la SRM, ne pas être des gens de l'Art, c'est-à-dire des médecins ou des pharmaciens. Si les questions relatives à cette évaluation nous sont au fond familières, pourquoi le travail de la SRM a-t-il été « oublié »? Outre l'attitude qui consiste à nier une valeur du passé, c'est en partie pour une raison en apparence anecdotique : après le décès de Vicq d'Azyr, le Comité de salut public avait décidé que les papiers manuscrits ou imprimés concernant l'art vétérinaire conservés à son domicile seraient remis à la Commission d'agriculture. Vicq d'Azyr resta donc pour la postérité le chantre de l'anatomie comparative et du rapprochement des médecines humaine et vétérinaire. Quant aux anciens de la SRM, ils ne revendiquèrent évidemment pas leur attachement à une compagnie royale. L'affirmation des nouvelles sociétés médicales passait par la négation ostensible, à défaut d'être effective, du passé ; mieux valait se prévaloir de réflexions et d'actions inédites afin de bénéficier d'une audience et donc d'être efficace.

Autre thème, celui du progrès, donc de l'évolution de la médecine: la période étudiée vit «le passage de la médecine hippocratique à la médecine clinique » tandis que l'évaluation des médicaments utilisait d'autres sciences, comme la chimie, qui permettait de mieux comprendre l'action des médicaments et donc d'inventer des spécialités. Le développement des spécialités a rendu d'autant plus nécessaire leur évaluation, et si notre hypothèse est juste, cette dernière, à son tour, explique l'amplification de ce développement. L'officialisation de ce développement a été lent: « $\varepsilon n 1831$, seules les spécialités suivantes avaient une existence légale : pilules de Belloste, grains de santé du docteur Franck, poudre d'Irroë, rob Boyveau Laffecteur, pommade anti-ophtalmique de la veuve Fournier, préparations anti-dartreuses de Kunckel, pâte Regnaud, eau de Botot, poudre de Sency ». Dans cette liste figurent deux spécialités qui avaient été approuvées par la SRM: les pilules de Belloste et le rob Laffecteur. L'évaluation de la SRM était donc correcte, avec les outils qui étaient à sa disposition, puisque des décennies plus tard, ces médicaments étaient reconnus officiellement.

Personne ne songe à nier que les progrès de la médecine s'enracinent dans des acquis pluriséculaires. On peut dès lors s'étonner, et regretter, que les responsables de l'organisation de la santé publique se soucient peu des travaux de leurs homologues des siècles passés.
L'histoire n'est certes pas un réservoir de solutions pour les problèmes de notre temps. Mais, à trop l'ignorer, on se condamne à ne penser ces problèmes qu'avec ses moyens propres. Or les traces que les acteurs de la santé au XVIII ${ }^{e}$ ont laissées de leur pensée et de leur action - traces dont la connaissance pourrait, à nos yeux, favoriser des progrès sensibles en termes d'organisation - dorment sous la poussière des archives ou sont ensevelies avec des sciences peu considérées. Dans un domaine où, en fin de compte, c'est l'homme, son bien-être, sa vie ou sa mort qui sont en jeu, il nous paraît que rien ne devrait être négligé de ce qui peut enrichir la réflexion des acteurs de la santé publique, et féconder leur action. $\diamond$

\section{SUMMARY}

In the era of the Royal Society of Medicine

In the May 2013 issue of medecine/sciences, the Rob Laffecteur complained about the constraints imposed by the Société Royale de Médecine in 1779 , bearing on the labelling of remedies. Though, he did take advantage of the evaluation from an advertising point of view; though in this prospect he diverted his evaluation's report, in order to present it in a flattering manner. The Société Royale de Médecine was founded in 1778; its mission was to cover everything that had to do with public healthcare. Active, age-old and competent, it was submitted to many of contemporary issues that we are facing nowadays in the matter of medicines' evaluation, which is based on a rigorous scientific evaluation, itself based on knowledge's state-of-the-art. $\diamond$

\section{REMERCIEMENTS}

Nous remercions Laure Coulombel, Jacques Haiech, Anne-Marie Moulin, Jean Navarro pour leur intérêt, leur soutien et leurs remarques.

\section{LIENS D'INTÉRÊT}

L'auteur déclare n'avoir aucun lien d'intérêt concernant les données publiées dans cet article.

\section{POUR EN SAVOIR PLUS}

Le texte reprend de larges extraits de la thèse de doctorat de Pascale Gramain.

Gramain P. Le monde du médicament à l'aube de l'ère industrielle : les enjeux de la prescription médicamenteuse de la fin du xviii ${ }^{i}$ au début du xix siècle. Thèse de doctorat, directeur Michel Morange, Université Paris 7, 1999.

Les citations en italique dans le texte sont toutes extraites des archives de la Société Royale de Médecine, conservées à l'Académie de médecine.

- AM Manuscrit 7. Procès-verbaux de la SRM, 1779.

- AM SRM 96 d109. Dossier Boyveau Laffecteur.

- AM Manuscrit 8. Procès-verbaux de la SRM, $1780 ; 29: 370$.

- AM Manuscrit 14. Registre contenant le jugement de la SRM sur les remèdes et les différentes préparations qui lui ont été présentées, mars 1781. 


\section{AUTRES DOCUMENTS À CONSULTER}

Santé publique et remèdes au XVIII siècle

Bouvet M. Un remède secret du XVIII siècle : le rob Boyveau-Laffecteur. Bulletin de la Société d'Histoire de la Pharmacie, $1923 ; 11: 264-72$.

Choquet A. L'évolution des salaires dans l'industrie pharmaceutique, de la loi du 21 germinal an XI à

1948. Paris : Thèse de pharmacie, 1948, 8T1 Paris-737.

Faligot. La question des remèdes secrets sous la Révolution et l'Empire. Paris : Occitania, 1924

Goubert et Lepetit. Médicalisation de la société française 1770-1830. Ontario, Historical Reflection

Press, 1982.

Hannaway C. Public welfare and the state in the eighteenth century France : the Société Royale de Médecine of Paris (1776-1793). Thèse PhD, Johns Hopkins (Baltimore), 1974.

Nicole-Genty G. Les dernières années de la SRM. Le Progrès médical $1937 ; 14^{e}$ année, $n^{\circ} 7$

(supplément illustré) : 49-57.

Peter JP. Médecine, épidémies et société en France à la fin du XVIII ${ }^{e}$ siècle, d'après les archives de

l'Académie de médecine. Revue d'Histoire Moderne et Contemporaine $1970 ; n^{\circ} 4,14^{e}$ série.

Ramsey M. Property rights and right to health : the regulation of secret remedies in France, 1789-1815.

In : Bynum WF, Porter R, eds. Medical fringe and medical orthodoxy 1750-1850. Croom Helm Publisher, 1987.

Roche D. Talents, raison et sacrifice : l'image du médecin des Lumières d'après les éloges de la SRM.

Annales ESC 1977 ; $5: 866-82$.

Villebrun Joseph. Un remède anti-syphilitique aux XVIII' et XIXe siècles : le rob de Laffecteur. Paris

Librairie Le François, 1939.

Zwiebel-Muller P, La société de l'école de médecine et la santé publique en France de 1801 à 1821.

Thèse de $3^{e}$ cycle sous la direction de M.D. Grmek, École des hautes études en sciences sociales, $1978: 65$.

\section{Mercure et syphilis}

Benabou EM. La maladie antisociale : le danger vénérien à Paris au XVIII siècle dans la France d'ancien régime. Société de Démogaphie Historique. Toulouse Privat: 1984

Sprengel K. Maladie syphilitique produite par la dégénescence de la lèpre. In Histoire de la médecine depuis son origine jusqu'au XIX siècle. Paris : Librairie J.B. Baillière, vol. 9, 1832

Tilles $\mathrm{G}$, Wallach D. Le traitement de la syphilis par le mercure. Une histoire thérapeutique exemplaire. Hist Sci Med 1996 ; t. XXX (4) : 501-10.

Tilles G. Pénicilline et syphilis : les sacrifiés de l'Alabama. La Recherche 2012 ; $n^{\circ} 466: 108$.

Trousseau A, Pidoux H. Traité de thérapéutique et de matière médicale, vol. 1 ; Paris : Béchet Jeune, 1841.

\section{RÉFÉRENCE}

1. Gramain $P$. Les essais cliniques du rob Laffecteur : un remède secret du XVIII ${ }^{e}$ siècle. Med Sci (Paris) 2013 ; 29 : 537-8.

\section{TIRÉS À PART}

P. Gramain

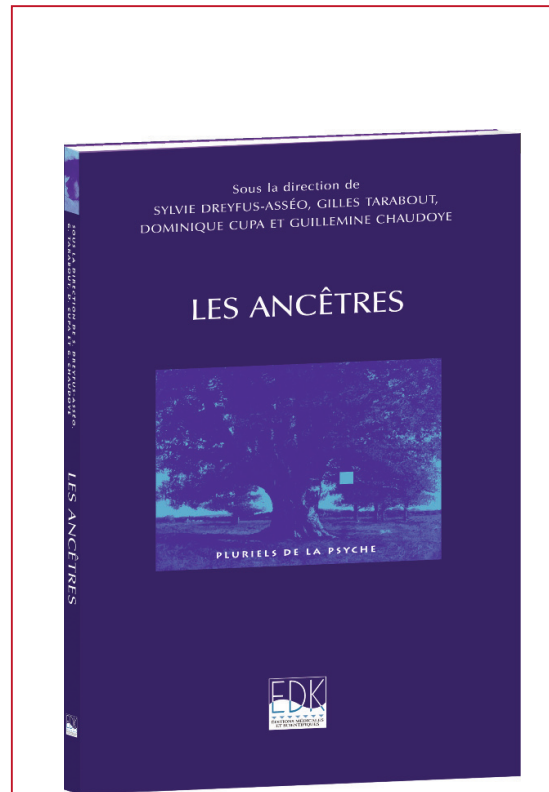

Prix : $17 €$

\section{LES ANCÊTRES}

Sous la direction de

S. Dreyfus-Asséo, G. Tarabout, D. Cupa et G. Chaudoye

Croisant leurs regards sur la question des Ancêtres, des psychanalystes et des anthropologues ont choisi de mener une réflexion commune autour de la transmission, de la filiation, de l'ancestralité et des processus d'ancestralisation.

Représentant d'une histoire individuelle, l'Ancêtre s'inscrit aussi dans une histoire collective, en tant que repère dans la filiation et contenant de la différence des générations. Point d'ancrage des processus d'identification, ni ombre, ni fantôme, l'Ancêtre se distingue du mort. Mort " revitalisé », il signe la capacité du sujet à investir sa propre histoire, à se l'approprier et à la partager. Loin d'être neutre et uniforme, l'ancestralité est une notion qui, de par les différences entre les sociétés, ouvre à une diversification des représentations, des croyances et des rituels. Idéal ou force de vie, relique ou fabriqué, l'Ancêtre ne peut se résumer à la question des origines et n'est pas seulement un héritage : il est le maillon d'un système dans lequel s'inscrit l'humain, il est ce « nouveau » issu d'un processus d'ancestralisation.

Titre : Les Ancêtres

Auteurs : Collectif Collection : Pluriels de la Psyché ISBN : 978-2-8425-4165-1

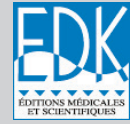

\section{Ouvrage disponible sur : www.edition-sciences.com}

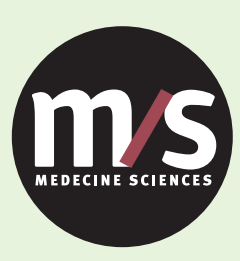

Tarifs d'abonnement $\mathrm{m} / \mathrm{s}-2013$

Abonnez-vous

à médecine/sciences
$>$ Grâce à $m / s$, vivez en direct

les progrès des sciences biologiques et médicales

\section{Bulletin d'abonnement} page 590 dans ce numéro de $\mathrm{m} / \mathrm{s}$

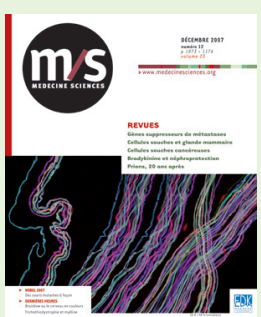

